\title{
Persistent eustachian valve
}

INSERM

\section{Source}

INSERM. (1999). Orphanet: an online rare disease and orphan drug data base. Persistent eustachian valve. ORPHA:99120

Persistent eustachian valve is a rare congenital anomaly of the inferior vena cava characterized by the postnatal presence of an eustachian valve remnant, which may be asymptomatic and considered a normal variant or prominent and clinically sig nificant. Clinical presentation is variable and includes obstruction of the inferior vena cava, cyanosis, thrombosis, pulmonary embolism, infective endocarditis, and when combined with persistent foramen ovale, it may generate permanent right-to-left shunt. 